\title{
PHENOTYPE ANALYSIS OF DIALLEL CROSSES
}

\author{
ALAN DURRANT \\ Department of Agricultural Botany, University College of Wales, Aberystwyth
}

Received 10.xii.68

\section{InTRODUGTION}

A PREVIOUS paper described methods of analysing reciprocal differences in diallel crosses (Durrant, 1965), hereafter referred to as paper I. The present paper gives a similar type of analysis for reciprocal means for the purpose of analysing: (1) dominance deviations due to reciprocal differences discussed in paper I ; (2) genetic differences determined by factors that are not necessarily mendelian, as for example, in crosses between flax genotrophs containing different amounts of nuclear DNA (Evans, Durrant, Rees, 1965); (3) phenotype patterns where no assumptions are made as to the frequencies, distributions or behaviour of genes; (4) non-genetic diallel tables.

In the analysis of genotype patterns the variation is formulated in terms of genetic components, e.g. Jinks' (1954), Hayman's (1954b) and Griffing's (1956) diallel analyses. Phenotype analyses examine phenotype patterns and give information on the behaviour of the parents on crossing in the generation or generations under consideration, but they vary in detail and complexity and can merge into genotype analyses. For example, part of Hayman's (1954a) analysis of variance of diallel crosses can be related to genotype patterns; Allard's analysis of variance of genotype/environment interactions is based on Hayman's (1954a) analysis. There are limitations, but on the other hand no assumptions need be made about gene distributions, frequencies, or types of interaction, the variation as a whole being regarded as that due to a biologically interacting system which may be split, for the purpose of defining patterns, into additive and interaction items in an analysis of variance. Like most analyses, phenotype analysis becomes more useful when applied to more extensive data, giving little genetic information on two parents and their $F_{1}$ but much more if they are grown in several environments, and/or crossed with other parents.

Phenotype analyses described here are appropriate for (1) and (4) above, probably also for (2), and worth trying on (3). The analyses are on the deviations of the $F_{1}$ values from their mid-parent values the purpose being to detect patterns corresponding to reciprocal differences patterns and which may therefore be due to reciprocal differences. The patterns are described in terms of alpha and beta inheritance, similar to those described in the analysis of reciprocal differences and cannot necessarily be identified with, or subdivided into, components of genetic variation.

Table $1(a)$ is a diallel table with $n=4$ parents where in the absence of reciprocal differences $y_{12}=y_{21}$, etc., or $y_{r s}=y_{s r}$. Other symbols are:

$P_{r}$ or $P_{s}=y_{r s}$ when $r=s$ (parents)

$P_{r}^{\prime}=P_{r}-\tilde{P}$

$V_{P}=$ variance of the parents

$W_{r \text { P }}=$ covariance of the female array of the $r$ th parent onto all parents.

$2 \mathrm{M} 2$ 
$W_{r \hat{\delta}}=$ covariance of the male array of the $r$ th parent onto all parents. $w_{r}=W_{r q}-W_{r 0^{*}}$

$w_{r}^{\prime}=w_{r}-\bar{w}$

$W_{r}=\frac{1}{2}\left(W_{r q}+W_{r^{\prime}}\right)$

$W_{r}^{\prime}=W_{r}-\bar{W}$

$g_{r s}=y_{r s}-y_{s r}$

Table $1(b)$ is a full table of $\mathrm{F}_{1}$ deviations from their midparent values where $c_{12}=c_{21}=\frac{1}{2}\left(y_{12}+y_{21}-P_{1}-P_{2}\right)$, or $c_{r 8}=c_{8 r}=\frac{1}{2}\left(y_{r 8}+y_{8 r}-P_{r}-P_{s}\right)$, and $c_{r s}=0$ when $r=s$. $C_{r}$ is the column and row total of the $r$ th parent and $W_{c r}$ is the covariance of the $c$ values of the $r$ th parent on to all parental

TABLE $1(a)$

Diallel table

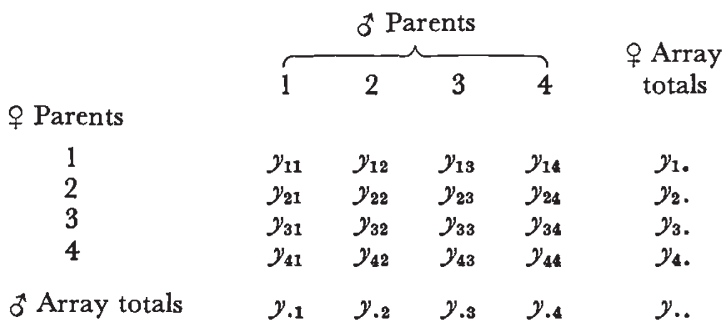

TABLE $1(b)$

Diallel table of dominance deviations

\begin{tabular}{cccccc}
\multicolumn{7}{c}{ Diallel table of dominance deviations } \\
Parents & 1 & 2 & 3 & 4 & Array \\
1 & $c_{11}$ & $c_{12}$ & $c_{13}$ & $c_{14}$ & $C_{1}$ \\
2 & $c_{21}$ & $c_{22}$ & $c_{23}$ & $c_{24}$ & $C_{2}$ \\
3 & $c_{31}$ & $c_{32}$ & $c_{33}$ & $c_{34}$ & $C_{3}$ \\
4 & $c_{41}$ & $c_{42}$ & $c_{43}$ & $c_{44}$ & $C_{4}$
\end{tabular}

values, $P$, including that of $c_{r s}$ when $r=s$ onto $P_{r}$. $W_{c r}$ is therefore the covariance of the deviations of the reciprocal means from their mid-parent values of the $r$ th parental array on to the non-recurrent parents. It can also be calculated using $W_{r}$, the covariance of the reciprocal means of the $r$ th parent onto the non-recurrent parents, from

$$
W_{c r}=W_{r}-\frac{1}{2} V_{P}
$$

\section{Alpha inheritance}

In the analysis of the reciprocal differences, alpha inheritance of any one parent measures the constant increase or decrease along its male or female array due to its maternal or paternal effect. In its estimation the alpha inheritance of the other parents along its reciprocal arrays is removed (paper I). Similarly, alpha inheritance of the reciprocal means occurs when a parent contributes a constant, or average, dominance deviation along its array of reciprocal means.

$c_{r s}$ is the balance of the dominance effects or potence (Wigan, 1944), for the $P_{r} \times P_{s}$ cross. If $a_{c r}$ is the dominance contribution of $P_{r}$ in each $\mathrm{F}_{\mathbf{1}}$ 
family having $P_{r}$ as one of its parents, and $a_{c s}$ is the dominance contribution of $P_{\delta}$ in each of its $F_{1}$ families, then the dominance deviation of the $P_{r} \times P_{\delta}$ cross expressed in terms of the individual parental contributions is

$$
c_{r s}=a_{c r}+a_{c s}
$$

and the dominance contribution of each parent to the diallel table is estimated from

$$
a_{c r}=\frac{2(n-1) C_{r}-\Sigma C}{2(n-1)(n-2)}
$$

and

$$
\bar{a}_{c}=\frac{1}{2 n(n-1)} \Sigma C .
$$

The $a_{c}$ values give the average amount of dominance, and its direction, contributed by each parent on crossing with the other parents. If $a_{c r}$ is positive, $P_{r}$ gives dominance deviations in a positive direction, $P_{r}$ being dominant if it has a large value, recessive if it has a small value. If two parents, $P_{r}$ and $P_{s}$, have $a_{c}$ values which sum to a greater value than $\frac{1}{2}\left(P_{r}-P_{s}\right)$ heterosis occurs when they are crossed. $2 \bar{a}_{c}$ is mean dominance, equal to the mean difference between parents and $F_{1} s$.

\section{TABLE 2}

\section{Alpha inheritance}

(a) Sums of squares

Item

S.S.

d.f.

Total

$\frac{1}{2} \Sigma c_{r 8}^{2}$

$2 \bar{a}_{c}$

$2(n-1) \frac{\Sigma^{2} a_{c}}{n}$

$\frac{1}{2} n(n-1)$

$a_{c}^{\prime}$

$(n-2)\left[\Sigma a_{c}^{2}-\frac{\Sigma^{2} a_{c}}{n}\right]$

1

Residual

By subtraction

$n-1$

Residual

$$
\frac{1}{2} n(n-3)
$$

(b) Array covariances

$$
\begin{array}{ll}
\bar{W} & \frac{1}{n-1}\left[\frac{1}{2} \Sigma P^{\prime 2}+\frac{n-2}{n} \Sigma a_{c} P^{\prime}\right] \\
W_{r}^{\prime} & \frac{2}{n-1}\left[\frac{1}{n} \Sigma a_{c} P^{\prime}-a_{c r} P_{r}^{\prime}\right] \\
\Sigma P^{\prime} W^{\prime} & -\frac{2}{n-1} \Sigma a_{c} P^{\prime 2}
\end{array}
$$

The sums of squares are given in table $2(a)$ where alpha is split into mean dominance, $2 \bar{a}_{c}$, and dominance deviations, $a_{c}$, of individual parents about mean dominance. The residual sum of squares measures all other dominance deviations which cannot be included in alpha. The three sums of squares are related respectively to the three sums of squares, $\left(b_{1}\right),\left(b_{2}\right),\left(b_{3}\right)$, of Hayman's (1954a) analysis. Both $2 \bar{a}_{c}$ and $\left(b_{1}\right)$ measure mean dominance but whereas $a_{c}^{\prime}$ and residual sums of squares contain the remaining dominance 
deviations, in Hayman's analysis some may be included in another item, $(a)$, the variation between mean effects of each parental line. The $(a)$ and $(b)$ symbols in brackets denoting Hayman's items are not the same as the $a$ and $b$ symbols used here for estimates of alpha and beta inheritance. Some tests of significance are given in section 5 .

The female array covariances, $W_{\text {}}$, plotted against the male array covariances, $W_{\delta}$, were used for interpreting reciprocal differences (paper $I$ ). In the absence of reciprocal differences and dominance all points are together at $W_{\odot}=W_{\delta^{*}}=\frac{1}{2} V_{P}$. Dominance deviations spread the points along a line of unit slope through the origin, reciprocal differences spread the points at right angles to this line, so that both can be studied together on the same graph (see section 5 ). The array covariance for the reciprocal means given in table $2(b)$ have been split to give a mean covariance, $\bar{W}$ of all arrays, and deviations of individual array covariances, $W_{r}^{\prime}$ from the mean. The more dominant parents are displaced towards the origin, i.e. when $a_{r}$ and $P_{r}$ are both large and positive, or both large and negative, and the more recessive parents away from the origin. $W_{r}=\bar{W}+W_{r}^{\prime}$ is the array covariance used by Jinks (1954) in the $W_{r} / V_{r}$ graph for examining the direction and distribution of dominant genes, and gene interaction. Consequently alpha inheritance spreads the points on the $W_{r} / V_{r}$ graph in the same general directions with regard to dominant and recessive parents as in his genotype analysis.

Array variances, $V_{r}$ formulated in terms of alpha give cumbersome expressions which make it difficult to interpret the slope given by alpha inheritance on the $W_{r} / V_{r}$ graph, although worked examples have given only small non-significant deviations from unit slope. When all $a_{c}$ values are equal giving a uniform dominance deviation, $2 \bar{a}_{c}$, over the whole table, due for example to some general interaction of parents and $F_{1} s$ with environments, the expressions reduce to

$$
W_{r}-W_{s}=V_{r}-V_{s}=\frac{2 \bar{a}_{c}\left(P_{s}-P_{r}\right)}{n-1}
$$

and the $W_{r} / V_{r}$ graph has unit slope with complete correlation between $W_{r}$, $V_{r}$ and $P_{r}$. The line cuts the $W_{r}$ axis at $\frac{1}{4}\left[V P-\frac{4}{n}\left(2 \tilde{a}_{c}\right)^{2}\right]$ which has an obvious resemblance to $\frac{1}{4}\left(D-H_{1}\right)$ of the biometrical model of Jinks and Hayman, but $2 \bar{a}_{c}$ measures mean dominance whereas $H_{1}$ takes into account as far as possible the direction of dominance of individual genes or effective factors provided there are no gene interactions and the genes are distributed at random.

\section{Beta inheritance}

In the analysis of reciprocal differences (paper I), beta inheritance of any one parent is a measure of the difference in dominance between its reciprocal arrays calculated from the regression of the reciprocal differences along its arrays on to the parents. This is the same as subtracting the regression of its male array onto the parents from that of the female array. In estimating the beta inheritance of one parent the beta inheritance of the other parents along its arrays is removed. Similarly, in the reciprocal means, beta inheritance of any one parent measures the dominance of the reciprocal means 
along its arrays calculated from the regression of the dominance deviations of the reciprocal means onto the parents. Since $W_{c r}=W_{r}-\frac{1}{2} V P$, the regression coefficients are related to the arrangement of points on the $W_{r} / V_{r}$ graph of Jinks (1954) except that they are adjusted to remove, for any one parent, the beta inheritance of the other parents along its array, and to make the regression line for the array of any one parent pass through the origin when $P_{r}=P_{s}$ i.e. a parent crossed with itself shows no dominance. The method may be approached via the potence ratio, as follows.

The average dominance ratio, or potence ratio (Wigan, 1944) of a cross between two parents, $P_{r}, P_{s}$, is the average dominance expressed as a fraction of half the parental difference,

$$
\frac{2 c_{r s}}{P_{r}-P_{s}}
$$

Beta inheritance measures the contributions of individual parents to the potence ratios over the whole table. The contribution of $P_{r}$ in crosses with other parents, $P_{s}$ is measured by $b_{c r}$ in

$$
2 c_{r s}=b_{c r}\left(P_{r}-P_{s}\right) \text {. }
$$

Taking into account the contribution of both parents of each cross and omitting the factor of 2 this becomes

$$
c_{r s}=\left(b_{c r}-b_{c s}\right)\left(P_{s}-P_{r}\right),
$$

which is beta inheritance. The least squares equations are

$$
\begin{aligned}
& b_{c_{1}}\left[\Sigma P^{\prime 2}+(n+1) P_{1}^{\prime 2}\right]+b_{c_{2}}\left(2 P_{1}^{\prime} P_{2}^{\prime}-P_{2}^{\prime 2}\right) \ldots b_{c n}\left(2 P_{1}^{\prime} P_{n}^{\prime}-P_{n}^{\prime 2}\right)= \\
& (n-1) W_{c_{1}}-P_{1}^{\prime} C_{1} \\
& b_{c_{1}}\left(2 P_{2}^{\prime} P_{1}^{\prime}-P_{1}^{\prime 2}\right)+b_{c_{2}}\left[\Sigma P^{\prime 2}+(n+1) P_{2}^{\prime 2}\right] \ldots b_{c n}\left(2 P_{2}^{\prime} P_{n}^{\prime}-P_{n}^{\prime 2}\right)= \\
& (n-1) W_{c_{2}}-P_{2}^{\prime} C_{2} \\
& b_{c_{1}}\left(2 P_{n}^{\prime} P_{1}^{\prime}-P_{1}^{\prime 2}\right)+b_{c_{2}}\left(2 P_{n}^{\prime} P_{2}^{\prime}-P_{2}^{\prime 2}\right) \ldots b_{c n}\left[\Sigma P^{\prime 2}+(n+1) P_{n}^{\prime 2}\right]= \\
& \text { and } \Sigma b_{c}=0 \text {. } \\
& (n-1) W_{c n}-P_{n}^{\prime} C_{n}
\end{aligned}
$$

The $b_{c}$ values give the relative dominance potentials ascribable to the different parents. Two parents with the same $b_{c}$ value have the same potential and when crossed would be expected to give an $F_{1}$ with midparent value. Parents with smaller positive, or larger negative, values are dominant over parents with the larger positive, or smaller negative, values. A difference of 0.5 between two $b_{c}$ values indicates that one parent is fully dominant over the other as assessed over the whole table. If two parents have $b_{c}$ values which differ by more than 0.5 , the cross between them is expected to show heterosis. The average direction of dominance is given by $\Sigma b_{c} P$, which is negative if average dominance is in a positive direction, and

$$
\Sigma_{c_{r s}}=-2 n \Sigma b_{c} P
$$

over the full table, so that the overall difference between parental and $F_{1}$ means is dependent upon the magnitude of the $b_{c}$ values and their correlation with $P$. The sum of squares is given in table $3(a)$, where the beta item 
includes the overall difference between parents and $\mathrm{F}_{1} \mathrm{~s}$; other comments are made in section 5 . As judged by the array covariances, $W_{r}=\bar{W}+W_{r}^{\prime}$, in

TABLE 3

\section{Beta inheritance}

(a) Sums of squares

Item

S.S.

d.f.

Total

$\frac{1}{2} \Sigma c_{r 8}^{2}$

$\frac{1}{2} n(n-1)$

Beta

$\Sigma b_{c r}\left[(n-1) W_{c r}-P_{r}^{\prime} C_{r}\right]$

$n-1$

Residual

By subtraction

$\frac{1}{2}(n-2)(n-1)$

(b) Array covariances

$\bar{W}$

$$
\frac{1}{n-1}\left[\frac{1}{2} \Sigma P^{\prime 2}-\Sigma b_{c} P^{\prime 2}\right]
$$

$W_{r}^{\prime} \quad \frac{1}{n-1}\left[b_{c r} \Sigma P^{\prime 2}+P_{r}^{\prime} \Sigma b_{c} P^{\prime}\right]$

$\Sigma P^{\prime} W^{\prime} \quad \frac{2}{n-1} \Sigma P^{\prime 2} \Sigma b_{c} P^{\prime}$

table $3(b)$, with beta inheritance the more dominant parents are again displaced towards the origin on the $W_{r} / V_{r}$ graph and the more recessive away from the origin. $\Sigma P_{r}^{\prime} W_{r}$ is also a measure of the overall direction of dominance, being negative for dominance in a positive direction.

TABLE 4

Alpha and beta inheritance

For explanation of $(a)-(f)$ see text

(a)

$\begin{array}{llll}2 & 3 & 4 & 5 \\ 3 & 4 & 5 & 6 \\ 4 & 5 & 6 & 7 \\ 5 & 6 & 7 & 8\end{array}$

(c)

$\begin{array}{llll}2 & 2 \cdot 5 & 3 & 3 \cdot 5 \\ 2 \cdot 5 & 4 & 5 & 6 \\ 3 & 5 & 6 & 7 \\ 3 \cdot 5 & 6 & 7 & 8\end{array}$

(e)

$\begin{array}{llll}2 & 4 & 3 & 2 \\ 4 & 4 & 5 & 6 \\ 3 & 5 & 6 & 7 \\ 2 & 6 & 7 & 8\end{array}$

(b)

$\begin{array}{llll}2 & 2 & 2 & 2 \\ 2 & 4 & 5 & 6 \\ 2 & 5 & 6 & 7 \\ 2 & 6 & 7 & 8\end{array}$

(d)

$\begin{array}{llll}2 & 4 & 6 & 8 \\ 4 & 4 & 5 & 6 \\ 6 & 5 & 6 & 7 \\ 8 & 6 & 7 & 8\end{array}$

$(f)$

\section{Alpha ANd beta inheritance}

Beta measures the dominance potentials of the parents, which are derived from the potence ratios. It also measures the increase in average dominance in a positive or negative direction, with increase in parental difference. Alpha, if estimated jointly with beta, may be used as an adjustment such that 
beta will also measure decrease in average dominance with increase in parental difference, and other patterns that cannot be measured by either separately. An illustration is given in table 4 . In $4(a)$ there is no dominance. $4(b),(c)$ and $(d)$ contain beta only; the first parent is fully dominant in $(b)$, in $(c)$ it is partially dominant and in $(d)$ it is fully recessive. In $4(e)$ the first parent is fully recessive to the second parent, shows partial dominance with the third and is fully dominant to the fourth. This pattern can be converted to beta by subtracting 5 units from each $F_{1}$ in the arrays of the first parent, i.e. subtracting an alpha component. In $4(f)$ the trend is the same as in $4(e)$ but it is less pronounced and can be converted to beta by subtracting 2 units. In the analysis the contributions of other parents are taken into account with the result that joint estimates of alpha and beta accommodate a large range of patterns. On the other hand beta may act as an adjustment to alpha for parents of more similar genetic constitution which give less dominance on crossing. Writing

$$
c_{r s}=a_{c r}+a_{c s}+\left(b_{c r}-b_{c s}\right)\left(P_{s}-P_{r}\right)
$$

the joint estimates for alpha and beta inheritance are obtained from the equations in table 5 . The sum of squares is

$$
\Sigma b_{c r}\left[(n-1) W_{c r}-P_{r}^{\prime} C_{r}\right]+\Sigma a_{c r} C_{r}
$$

with $2 n-1$ degrees of freedom.

\section{TABLE 5}

Foint estimation of alpha and beta

$$
\begin{aligned}
& b_{c 1}\left[\Sigma P^{\prime 2}+(n+1) P_{1}^{\prime 2}\right]+b_{c 2}\left(2 P_{1}^{\prime} P_{2}^{\prime}-P_{2}^{\prime 2}\right) \ldots b_{c n}\left(2 P_{1}^{\prime} P_{n}^{\prime}-P_{n}^{\prime 2}\right)-a_{c 1} n P_{1}^{\prime}+a_{c 2}\left(P_{2}^{\prime}-P_{1}^{\prime}\right) \ldots \\
& a_{c n}\left(P_{n}^{\prime}-P_{1}^{\prime}\right)=(n-1) W_{c 1}-P_{1}^{\prime} C_{1} \\
& b_{c 1}\left(2 P_{2}^{\prime} P_{1}^{\prime}-P_{1}^{\prime 2}\right)+b_{c 2}\left[\Sigma P^{\prime 2}+(n+1) P_{2}^{\prime 2}\right] \ldots b_{c n}\left(2 P_{2}^{\prime} P_{n}^{\prime}-P_{n}^{\prime 2}\right)+a_{c 1}\left(P_{1}^{\prime}-P_{2}^{\prime}\right)-a_{c 2} n P_{2}^{\prime} \cdots \\
& a_{c n}\left(P_{n}^{\prime}-P_{2}^{\prime}\right)=(n-1) W_{c 2}-P_{2}^{\prime} C_{2} \\
& b_{c 1}\left(2 P_{n}^{\prime} P_{1}^{\prime}-P_{1}^{\prime 2}\right)+b_{c 2}\left(2 P_{n}^{\prime} P_{2}^{\prime}-P_{2}^{\prime 2}\right) \ldots b_{c n}\left[\Sigma P^{\prime 2}+(n+1) P_{n}^{\prime 2}\right]+a_{c 1}\left(P_{1}^{\prime}-P_{n}^{\prime}\right)+a_{c 2}\left(P_{2}^{\prime}-P_{n}^{\prime}\right) \ldots \\
& -a_{c n} n P_{n}^{\prime}=(n-1) W_{c n}-P_{n}^{\prime} C_{n}
\end{aligned}
$$

$-b_{c 1}(n+1) P_{1}^{\prime}-b_{c 2} P_{2}^{\prime} \ldots-b_{c n} P_{n}^{\prime}+a_{c 1}(n-1)+a_{c 2} \cdots+a_{c n}=C_{1}$

$-b_{c 1} P_{1}^{\prime}-b_{c 2}(n+1) P_{2}^{\prime} \ldots-b_{c n} P_{n}^{\prime}+a_{c 1}+a_{c 2}(n-1) \ldots a_{c n}=C_{2}$

$-b_{c 1} P_{1}^{\prime}-b_{c 2} \bar{P}_{2}^{\prime} \ldots-b_{c n}(n+1) P_{n}^{\prime}+a_{c 1}+a_{c 2} \ldots a_{c n}(n-1)=C_{n}$

Beta inheritance superimposed on a uniform dominance increment, $2 \bar{a}_{c}$ over the whole table where

$$
c_{r s}=2 \vec{a}_{c}+\left(b_{c r}-b_{c s}\right)\left(P_{s}-P_{r}\right)
$$

is estimated from the equations in table 6. The sum of squares is

$$
\Sigma b_{c r}\left[(n-1) W_{c r}-P_{r}^{\prime} C_{r}\right]+\bar{a} \Sigma C
$$

with $n$ degrees of freedom. 
Genically, gene dispersion giving approximately equal parental values could be usefully analysed with alpha. Beta gives an exact fit for extreme gene association; if a major gene were responsible for most of the variation beta would be correspondingly large.

\section{TABLE 6}

Joint estimation of beta and $\overline{\mathbf{a}}_{\mathbf{c}}$

$$
\begin{aligned}
& b_{c 1}\left[\Sigma P^{\prime 2}+(n+1) P_{1}^{\prime 2}\right]+b_{c 2}\left(2 P_{1}^{\prime} P_{2}^{\prime}-P_{2}^{\prime 2}\right) \ldots b_{c n}\left(2 P_{1}^{\prime} P_{n}^{\prime}-P_{n}^{\prime 2}\right)-\bar{a}_{c} 2 n P_{1}^{\prime}=(n-1) W_{c 1}-P_{1}^{\prime} C_{1} \\
& b_{c 1}\left(2 P_{2}^{\prime} P_{1}^{\prime}-P_{1}^{\prime 2}\right)+b_{c 2}\left[\Sigma P^{\prime 2}+(n+1) P_{2}^{\prime 2}\right] \ldots b_{c n}\left(2 P_{2}^{\prime} P_{n}^{\prime}-P_{n}^{\prime 2}\right)-\bar{a}_{c} 2 n P_{2}^{\prime}=(n-1) W_{c 2}-P_{2}^{\prime} C_{2} \\
& b_{c 1}\left(2 P_{n}^{\prime} P_{1}^{\prime}-P_{1}^{\prime 2}\right)+b_{c 2}\left(2 P_{n}^{\prime} P_{2}^{\prime}-P_{2}^{\prime 2}\right) \ldots b_{c n}\left[\Sigma P^{\prime 2}+(n+1) P_{n}^{\prime 2}\right]-\bar{a}_{c} 2 n P_{n}^{\prime}=(n-1) W_{c n}-P_{n}^{\prime} C_{n} \\
& -b_{c 1} 2 n P_{1}^{\prime}-b_{c 2} 2 n P_{2}^{\prime} \cdots-b_{c n} 2 n P_{n}^{\prime}+\bar{a}_{c} 2 n(n-1)=\Sigma C
\end{aligned}
$$

\section{REGipRocal DifFERENGES AND MEANS}

Reciprocals of a particular cross deviating by equal amounts in opposite directions from the mid-parent value are said to be symmetrical (paper I). If one reciprocal difference has the mid-parent value and the other deviates in either direction the reciprocal difference is said to be asymmetric. Reciprocal differences between these two forms are partially asymmetric.

Reciprocal differences were measured in terms of alpha and beta inheritance (paper I) and their estimates are denoted here as $a_{g}$ and $b_{g}$ respectively. Combining formulae for reciprocal differences and reciprocal means,

$$
y_{r s}=\frac{1}{2}\left(P_{r}+P_{s}\right)+c_{r s}+\frac{1}{2} g_{r s} .
$$

The alpha contribution of female parent $P_{r q}$ to dominance is

$$
a_{r \text { }}=a_{c r}+\frac{1}{2} a_{g r}
$$

and that of the male parent, $P_{r \sigma^{*}}$ is

$$
a_{r \delta}=a_{c r}-\frac{1}{2} a_{g r} \text {. }
$$

Similarly, the beta contributions are

$$
\begin{aligned}
& b_{r ף}=b_{c r}+\frac{1}{2} b_{g r} . \\
& b_{r \sigma^{*}}=b_{c r}-\frac{1}{2} b_{g r} .
\end{aligned}
$$

If dominance deviations are due entirely to asymmetric reciprocal differences, and the factors determining the reciprocal differences influence the female arrays only,

$$
b_{g r}-b_{g}=2 b_{c r}
$$

and the male arrays only

$$
b_{g r}-b_{g}=-2 b_{c r} \text {. }
$$

Therefore female array effects give a regression coefficient of 0.5 for $b_{c}$ on $b_{g}$, and -0.5 for male array effects. If the reciprocal differences are partly 
asymmetric the slope is reduced and if there is no consistent measure of asymmetry due to either male, or female, array effects, or the dominance deviations are due to other causes than reciprocal differences, there will be no significant regression. $\quad b_{c}=0$ and is thus unaffected by $\bar{b}_{g}$.

If alpha inheritance of the reciprocal differences influences the respective parents by the same amount as it does the crosses, it will have no effect on the reciprocal means which will have mid-parent values and all $a_{c}$ values will be zero if there is no other component of dominance present. If it is asymmetric, or partly asymmetric, and it does not influence the parents by the same amount as the crosses the reciprocal means will also show alpha inheritance. If it is asymmetric and the parents are not influenced, if the female arrays are increased or decreased,

$$
a_{g r}=2\left(a_{c r}-\bar{a}_{c}\right)
$$

and if the male arrays are increased or decreased

$$
a_{g r}=-2\left(a_{c r}-\bar{a}_{c}\right)
$$

the regression coefficient of $a_{c}$ on $a_{g}$ being 0.5 and -0.5 respectively for female and male array effects as before. It follows that with alpha reciprocal differences, if there are no dominance deviations then either alpha influences the parents and crosses alike, or it is symmetrical, but if there is a significant regression of $a_{c}$ on $a_{g}$ alpha is asymmetric, or partially asymmetric, and either does not influence the parents at all or does so to a greater, or lesser, extent than it does the crosses. $\vec{a}_{g}=0$ because $a_{g}$ values measure the relative magnitudes of the reciprocal differences, but $\bar{a}_{c}$ may be determined by the $a_{g}$ values depending on whether they consistently add to, or subtract from, the maternal or paternal arrays, so that an estimated value, $\hat{\bar{a}_{g}}$, could be obtained from

$$
\hat{\bar{a}}_{g}=2 \bar{a}_{c} .
$$

If alpha and beta occur together in the reciprocal differences, the above relations do not necessarily hold, particularly with regard to the inflation of the regression coefficients which may occur for several reasons. First, erratic deviations may inflate the $a_{c}$ and $b_{c}$ values. Second, alpha and beta of the reciprocal differences are redistributed about a common mean $\bar{b}_{g}$. Third, $b_{g}$ values correlated with the parental values are split into two parts in the joint analysis; one part, due to asymmetry of parents, is transferred to $b_{g}$ and the other part is transferred to alpha, contributing $a_{g}$ values, $a_{g k}-a_{g k}{ }^{\prime}$, not correlated with the parental values. This second part gives a regression coefficient of $\frac{n}{2(n-2)}$ for $a_{c}$ on $a_{g}$ for female array effects, negative for male array effects, and is always greater than \pm 0.5 , but it does not give an exact fit, in terms of $a_{c}$ values, to the resultant dominance deviations. The first part can also be transferred to alpha if required but its contributed $a_{g}$ values, $a_{g k}{ }^{\prime}$, are correlated with the parent values.

The contributions of correlated beta to alpha are calculated as follows. If $k$ is the regression coefficient of $b_{g}$ on $P$ estimated from the beta analysis only, $a_{g r}$ values contributed by the whole of correlated beta are

$$
a_{g r k}=k\left[P_{r}^{\prime 2}-\frac{1}{n} \Sigma P^{\prime 2}\right],
$$


which are separated into a part correlated with the parents,

$$
a_{g r k^{\prime}}=\frac{k P_{r}^{\prime} \Sigma P^{\prime 3}}{\Sigma P^{\prime 2}}
$$

and a part not correlated,

$$
a_{g r k}-a_{g r k} k^{\prime}=k\left[P_{r}^{\prime 2}-\frac{1}{n} \Sigma P^{\prime 2}-\frac{P_{r}^{\prime} \Sigma P^{\prime 3}}{\Sigma P^{\prime 2}}\right] .
$$

In the same way that reciprocal differences in paternal directions do not necessarily imply paternal effects (paper I), a negative $a_{c} / a_{g}$ or $b_{c} / b_{g}$ correlation does not necessarily mean that the paternal array effects are paternal in origin for the above relations are based upon the assumption that the dominance deviations would be in the same direction as the asymmetric, or partially asymmetric, reciprocal differences which caused them. The correlation would be negative for example where the maternal environment of a small species was sub-optimal for the proper development of heterotic $F_{1}$ families given by crosses with other parents.

Dominance deviations of the reciprocal means shift the points on the $W_{\odot} / W_{\text {。 }}$ graph along a line of unit slope, reciprocal differences shift them at right angles to this line. It is necessary to determine whether comparable changes in reciprocal differences and dominance deviations are recorded to the same extent by the graph. The point, $W_{r+\%}, W_{r \sigma^{*}}$, may also be located by $\sqrt{2} W_{r}$ from the origin along the line of unit slope and at $\sqrt{\frac{1}{2}} w_{r}$ at right angles to the line of unit slope. For comparative purposes it will be useful to assume that all dominance deviations are due to asymmetric alpha or beta reciprocal differences and since $b_{c}$ or $a_{c}$ is only half as much as $b_{g}$ or $a_{g}$ the expressions can be written as $\sqrt{\frac{1}{2}} W_{r}$ and $\sqrt{\frac{1}{2}} w_{r}$ respectively, or for comparative purposes simply $W_{r}$ and $w_{r}$ and these are given in table 7 ; (i), in the absence of alpha and beta; (ii) to (v), for beta; and (vi) to (ix), for alpha.

Taking beta, the average position of the points, $\bar{W}, \bar{w}$, is given in (ii). The variation about the average position in each direction is expressed as a variance in (iii) to (v). The spread of points along a line of unit slope is in general much greater than at right angles to it except when

$$
\Sigma b_{c} P^{\prime}=\Sigma b_{g} P^{\prime}=0
$$

i.e. when there is no correlation between $b$ and $P$, and the two variances are equal. When completely correlated, and writing $b_{g}=k P^{\prime}$ in $(\mathrm{v})$, the points are spread out along a line of unit slope, as deduced by another method in paper I, the displacement of the line from the origin being dependent upon (ii). Thus with asymmetric beta inheritance the spread of the points along the line of unit slope is generally greater than at right angles to it.

Turning to alpha, both $\bar{W}$ and $\bar{w}$ are dependent upon the correlation of $a$ with $P$ but, as demonstrated in paper I, $a_{g}$ makes no contribution to $V_{w}$ so that all points are dispersed along the line of unit slope only. Thus the $W_{\odot} / W_{\text {s }}$ graph gives greater weight to dominance deviations than to reciprocal differences, and a smaller scatter about the line of unit slope than along it does not necessarily mean that reciprocal differences are smaller than dominance deviations which could themselves be due to the reciprocal differences. The interpretation of the distribution of the points along a line of unit slope in the $W_{\uparrow} / W_{\delta}$ graph given in the previous sections in terms of 
alpha and beta, and dominant and recessive parents, is in general the same as the interpretation of points on the $W_{r} / V_{r}$ graph of Jinks (1954) and Hayman (1954). Contributions will also be made to the $W_{r} / V_{r}$ graph by asymmetric or partially asymmetric reciprocal differences.

TABLE 7

Comparison of reciprocal means and differences on the $W_{\phi} / W_{\text {ơ }}$ graph

For explanation of (i)-(ix) see text

$W$

(i) Alpha and beta absent $\frac{1}{2(n-1)} \Sigma P^{\prime 2}$

(ii) Beta; mean

$\frac{1}{n-1}\left[\frac{1}{2} \Sigma P^{\prime 2}-\Sigma b_{c} P^{\prime}\right]^{2}$

$\frac{1}{(n-1)}\left[b_{g} \Sigma P^{\prime 2}+\Sigma b_{\theta} P^{\prime 2}\right]$

(iii) Beta; variance

$\frac{1}{(n-1)^{3}}\left[\Sigma b_{c}^{2} \Sigma^{2} P^{\prime 2}+3 \Sigma^{2} b_{c} P \Sigma P^{\prime 2}\right] \frac{1}{(n-1)}\left[\Sigma\left(b_{o}-b_{q}\right)^{2} \Sigma^{2} P^{\prime 2}-\Sigma^{2} b_{g} P^{\prime} \Sigma P^{\prime 2}\right]$

(iv) Beta; variance

$$
\frac{1}{(n-1)^{3}} \Sigma b_{c}^{2} \Sigma^{2} P^{\prime 2} \quad \frac{1}{(n-1)} \Sigma\left(b_{g}-b_{g}\right)^{2} \Sigma^{2} P^{\prime 2}
$$

$$
\begin{aligned}
& \Sigma b_{c} P^{\prime}=0 \\
& \Sigma b_{g} P^{\prime}=0
\end{aligned}
$$

(v) Beta; variance

$$
\frac{1}{(n-1)^{3}} k^{2} \Sigma^{2} P^{\prime 2}
$$

$$
\begin{aligned}
& b_{\text {cr }}=\frac{1}{2} k P_{r}^{\prime} \\
& b_{g r}=k P r
\end{aligned}
$$

(vi) Alpha; mean

$$
\begin{aligned}
& \frac{1}{(n-1)}\left[\frac{1}{2} \Sigma P^{\prime 2}+\frac{n-2}{n} \Sigma a_{c} P^{\prime}\right] \\
& \frac{4}{(n-1)^{3}}\left[\Sigma a_{c}^{2} P^{\prime 2}-\frac{1}{n} \Sigma^{2} a_{c} P^{\prime}\right] \\
& \frac{4}{(n-1)^{3}} \Sigma a_{c}^{2} P^{\prime 2}
\end{aligned}
$$$$
\frac{1}{(n-1)} \Sigma a_{o} P^{\prime}
$$

(vii) Alpha; variance

$$
\begin{aligned}
& \Sigma a_{c} P^{\prime}=0 \\
& \Sigma a_{g} P^{\prime}=0
\end{aligned}
$$

(ix) Alpha; variance

$$
\begin{aligned}
& a_{c r}=\frac{1}{2} k^{\prime} P^{\prime} \\
& a_{g r}=k^{\prime} P^{\prime}
\end{aligned}
$$$$
\frac{1}{(n-1)^{3}}\left[k^{\prime 2} \Sigma P^{\prime 4}-\frac{2 k^{\prime}}{n} \Sigma^{2} P^{\prime 2}\right]
$$

$b_{g}^{\prime}$ values are dependent upon $w=W_{\uparrow}-W_{\delta}$ and measures dominance changes in the male and female arrays in the same way as changes in positions of parents on the $W_{r} / V_{r}$ graph are interpreted as changes in dominance in different environments. In paper $I$ it was shown that

$$
-2 \vec{b}_{g}=\frac{\Sigma a_{g} P^{\prime}}{\Sigma P^{\prime 2}}
$$

where mean beta inheritance in the reciprocal differences could be interpreted as alpha inheritance correlated with the parental values. Genetic differences between the two interpretations appear in the reciprocal means. For example, if all $F_{1}$ families have the same values as their female parents, i.e. extreme maternal inheritance, $\bar{b}_{g}=-0.5$ (uniform beta) and all reciprocal means have mid-parent values (no dominance). There is no way 
of adding or subtracting the estimated $a_{g}$ values derived from the above equation, although giving an exact fit to the reciprocal differences, to obtain the original table of maternal effects, nor the same table of reciprocal means, apart from the unlikely situation where half $a_{g}$ values were added and subtracted respectively to rows and from columns. It may therefore be possible to establish whether $b_{g}$ is due to uniform beta or correlated alpha since there would be no correlation between $a_{g}$ and $a_{c}$ values for uniform beta but there would be for correlated alpha, unless the parents are influenced by alpha to the same extent when the situation would be indistinguishable from uniform beta.

Independent estimates of alpha and beta cannot be obtained when they occur together in the analysis of either reciprocal means or differences, and it is not possible to obtain a complete separation of the estimates in the reciprocal differences where, too, the influence of alpha on the parental values may cause difficulties which may be reflected in the analyses of means and differences. A partial separation of alpha and beta in the reciprocal differences was given in paper I where the sums of squares and products were set out and compared. A more critical test of significance for means and differences is to determine whether the joint mean square for alpha and beta combined takes out a significantly greater amount of variation than does the alpha, or beta, mean square obtained in the analysis assuming only alpha, or beta, present.

In genetic diallels reciprocal differences are measured independently of the parental values whereas dominance deviations are dependent upon them. In competition diallels the measurement of both means and differences is dependent on the parental values. The analyses in this and paper I are essentially regression analyses where the parents are assumed to have fixed values. "Competition" in competition diallels is estimated by McGilchrist (1965) in the same way as alpha inheritance is estimated here, giving tests of significance which include an item equivalent to parental error variation. Transposed to these symbols, and using the appropriate residual variation, $V_{e g}$ for reciprocal differences, $V_{e c}$ for reciprocal means, plus the error variation of the parents, $V_{e P}$, the error mean square for testing the $2 \vec{a}_{c}$ mean square in table $2(a)$ is $V_{e c}+(n-1) V_{e P}$, and for $a_{c}^{\prime}$ it is $V_{e c}+\frac{n-2}{2} V_{e P}$. For testing $a_{g}, V_{e g}$ is appropriate for genetic diallels but $V_{e g}+\frac{n}{2} V_{e P}$ should be used for competition diallels. The use of the residual variation for $V_{e}$ allows tests for uniformity of alpha or beta along arrays whereas a single large dominance deviation may give significance using the error from replication. It is often the case that the parents can be obtained and grown in larger numbers more easily than the $\mathrm{F}_{1} \mathrm{~s}$ of crosses between them since crossing within the parental lines may be unnecessary, thereby reducing the parental error variation to a relatively small amount. Twice the number of individuals for the parents are frequently used in diallels. The alpha and beta estimates may also be tested for uniformity over replicates.

\section{EXAMPLES}

For illustrative purposes, Jinks' and Broadhurst's data (1963) on rat weight at birth and 100 days will be used (the reciprocal differences were 
analysed in paper $\mathrm{I}$ ), and $\mathrm{F}_{1}$ and $\mathrm{F}_{2}$ data of a diallel cross of flax genotrophs and genotypes (Durrant and Tyson, 1964). Another analysis in paper I on a Nicotiana diallel recorded by Jinks and presented by Hayman (1954a) gave alpha inheritance in the reciprocal differences and it was concluded that this was probably due to the arrangement of the families in the greenhouse. The $a_{g}$ values were $-0 \cdot 1,-2 \cdot 4,-7 \cdot 1,9 \cdot 4,3 \cdot 1,3 \cdot 4,-1 \cdot 8,-4 \cdot 5$. The $a_{c}$ values are $-3 \cdot 3,-5 \cdot 2,-1 \cdot 0,-0.5,0.5,-3 \cdot 5,-1 \cdot 4,-1 \cdot 9$. The regression coefficient of $a_{g} / a_{c}$ is 0.24 , but the correlation coefficient is only 0.08 , i.e. dominance deviations are not directly and uniformly affected by the greenhouse arrangement which is to be expected since it is impossible to have one greenhouse effect to operate only on the female arrays and the other on the male arrays.

(i) $\mathrm{F}_{1}$ and $\mathrm{F}_{2}$ plant weights in a diallel cross between flax genotypes and genotrophs (table 8)

Reciprocal means only have been analysed since the reciprocal differences are small and the $W_{q} / W_{\delta}$ graphs give very little scatter from the line of unit

TABLE 8

$F_{1}$ and $F_{2}$ mean plant weights (in that order) $P, M$, are flax genotypes; $R, D$, are linseed genotypes; $S, L$, are flax genotrophs. $\left(\mathrm{F}_{1}\right.$, means of two replicates; $F_{2}$, means of six replicates.)

\begin{tabular}{|c|c|c|c|c|c|}
\hline$P$ & $M$ & $R$ & $D$ & $S$ & $L$ \\
\hline $\begin{array}{l}68 \\
70\end{array}$ & $\begin{array}{l}59 \\
54\end{array}$ & $\begin{array}{l}110 \\
108\end{array}$ & $\begin{array}{l}89 \\
88\end{array}$ & $\begin{array}{l}63 \\
70\end{array}$ & $\begin{array}{l}80 \\
81\end{array}$ \\
\hline 52 & 31 & 98 & 79 & 35 & 39 \\
\hline & & & 39 & & \\
\hline 130 & 114 & 121 & 146 & 89 & 105 \\
\hline 97 & 87 & 107 & 105 & 85 & 104 \\
\hline 100 & 69 & 146 & 91 & 66 & 97 \\
\hline 77 & 64 & 112 & 87 & 70 & 83 \\
\hline 59 & 25 & 93 & 67 & 27 & 54 \\
\hline 60 & 47 & 92 & 63 & 36 & 59 \\
\hline 91 & 41 & 83 & 120 & 57 & 61 \\
\hline 77 & 57 & 103 & 92 & 59 & 73 \\
\hline
\end{tabular}

slope. The alpha and beta estimates are given in tables 9, 10, and the analyses of variance in table 11 . In the $F_{1}, 2 \bar{a}_{c}$ only is significant. In the $\mathrm{F}_{2}$, where $2 \bar{a}_{c}$ has even greater significance, beta is only marginally significant, and in the joint analyses, although they are themselves highly significant, beta does not take out a significant amount of variation over and above that taken out by $2 \bar{a}^{\prime}$ or alpha as a whole. Therefore dominance deviations are best interpreted in terms of alpha inheritance, in the $F_{1}$ and $F_{2}$, which is almost entirely due to the mean dominance deviation $2 \bar{a}_{c}$ which in the $\mathrm{F}_{2}$ is half that in the $\mathbf{F}_{1}$. The $2 \bar{a}_{c}$ values are also significant using the critical test.

There is a high correlation between means and variances of the parental 
TABle 9

$F_{1}$ of Linum diallel; estimates

\begin{tabular}{|c|c|c|c|c|c|c|c|}
\hline \multirow[b]{2}{*}{ Array } & \multirow[b]{2}{*}{$a_{0}$} & \multirow[b]{2}{*}{$b_{c}$} & \multicolumn{2}{|c|}{ Joint } & \multicolumn{2}{|c|}{ Joint } & \multirow{2}{*}{$\begin{array}{c}\log \\
a_{c} \times 10^{3}\end{array}$} \\
\hline & & & $2 \bar{a}_{c}$ & $b_{c}$ & $a_{c}$ & $b_{c}$ & \\
\hline$P$ & 8.7 & 0.060 & - & 0.084 & $9 \cdot 1$ & -0.008 & $56 \cdot 3$ \\
\hline$M$ & -0.5 & $0 \cdot 115$ & - & -0.007 & $-14 \cdot 3$ & $0 \cdot 265$ & $20 \cdot 0$ \\
\hline$R$ & $17 \cdot 3$ & -0.216 & - & -0.099 & $-3 \cdot 1$ & -0.255 & 96.9 \\
\hline$D$ & $15 \cdot 5$ & -0.082 & - & 0.049 & $25 \cdot 3$ & 0.270 & $84 \cdot 4$ \\
\hline$S$ & $1 \cdot 1$ & 0.061 & - & -0.061 & $14 \cdot 5$ & -0.219 & $65 \cdot 6$ \\
\hline$L$ & $4 \cdot 0$ & 0.063 & - & 0.033 & 4.8 & -0.052 & 16.9 \\
\hline $2 \bar{a}_{c}$ & $15 \cdot 4$ & - & $15 \cdot 7$ & - & $12 \cdot 2$ & - & 113.4 \\
\hline
\end{tabular}

TABLE 10

$F_{2}$ of Linum diallel; estimates

\begin{tabular}{|c|c|c|c|c|c|c|c|}
\hline \multirow[b]{2}{*}{ Array } & \multirow[b]{2}{*}{$a_{c}$} & \multirow[b]{2}{*}{$b_{c}$} & & & \multirow[b]{2}{*}{$a_{c} \times 10^{3}$} \\
\hline & & & $2 \bar{a}_{c}$ & $b_{c}$ & $a_{c}$ & $b_{c}$ & \\
\hline$P$ & $4 \cdot 0$ & 0.008 & - & 0.009 & $4 \cdot 0$ & -0.001 & $21 \cdot 0$ \\
\hline$M$ & $-1 \cdot 1$ & -0.013 & - & -0.098 & 1.0 & -0.046 & $22 \cdot 7$ \\
\hline$R$ & $10 \cdot 4$ & $-0 \cdot 186$ & - & -0.112 & $6 \cdot 5$ & -0.067 & $64 \cdot 9$ \\
\hline$D$ & 1.0 & 0.013 & - & 0.087 & $2 \cdot 5$ & 0.055 & $6 \cdot 4$ \\
\hline$S$ & 5.6 & $0 \cdot 121$ & - & 0.037 & $6 \cdot 0$ & -0.007 & 61.6 \\
\hline$L$ & $3 \cdot 1$ & 0.057 & - & 0.078 & $3 \cdot 6$ & $0 \cdot 066$ & $16 \cdot 0$ \\
\hline $2 \bar{a}_{c}$ & $7 \cdot 6$ & - & $7 \cdot 4$ & - & $7 \cdot 8$ & - & $64 \cdot 2$ \\
\hline
\end{tabular}

Table 11

Linum diallel; analysis of variance

(v) Log plant weight, mean squares $\times 10^{3}$

\begin{tabular}{|c|c|c|c|c|c|}
\hline \multirow[b]{2}{*}{ Item } & \multirow[b]{2}{*}{ d.f. } & \multicolumn{2}{|c|}{$F_{1}$} & \multicolumn{2}{|c|}{$\mathrm{F}_{2}$} \\
\hline & & $\begin{array}{l}\text { Mean } \\
\text { square }\end{array}$ & V.R. & $\begin{array}{l}\text { Mean } \\
\text { square }\end{array}$ & V.R. \\
\hline 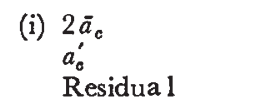 & $\begin{array}{l}1 \\
5 \\
9\end{array}$ & $\begin{array}{r}3542 \cdot 0 \\
222 \cdot 8 \\
124 \cdot 2\end{array}$ & $\begin{array}{c}28 \cdot 5 * * * \\
1.8 \\
-\end{array}$ & $\begin{array}{r}5290 \cdot 0 \\
380 \cdot 4 \\
33 \cdot 0\end{array}$ & $\begin{array}{c}160 \cdot 3^{* * *} \\
11 \cdot 5 \\
-\end{array}$ \\
\hline $\begin{array}{l}\text { (ii) Beta } \\
\text { Residual }\end{array}$ & $\begin{array}{r}5 \\
10\end{array}$ & $\begin{array}{l}467 \cdot 3 \\
344 \cdot 7\end{array}$ & $1 \cdot 4$ & $\begin{array}{l}976 \cdot 8 \\
260 \cdot 4\end{array}$ & $\begin{array}{l}3 \cdot 7^{*} \\
-\end{array}$ \\
\hline $\begin{array}{l}\text { (iii) Beta plus } 2 \bar{a}_{c} \\
\text { Residual }\end{array}$ & $\begin{array}{l}6 \\
9\end{array}$ & $\begin{array}{l}652 \cdot 7 \\
207 \cdot 4\end{array}$ & $3 \cdot 2$ & $\begin{array}{r}1214 \cdot 4 \\
22 \cdot 8\end{array}$ & $\begin{array}{l}53 \cdot 2 * * * \\
-\end{array}$ \\
\hline $\begin{array}{l}\text { (iv) Alpha plus beta } \\
\text { Residual }\end{array}$ & $\begin{array}{r}11 \\
4\end{array}$ & $\begin{array}{l}466 \cdot 7 \\
162 \cdot 3\end{array}$ & $\stackrel{2 \cdot 9}{-}$ & $\begin{array}{r}667 \cdot 2 \\
37 \cdot 2\end{array}$ & $17 \cdot 9 * *$ \\
\hline $\begin{array}{l}\text { (v) Log plant weight } \\
2 \bar{a}_{c} \\
a_{o}^{\prime} \\
\text { Residual }\end{array}$ & $\begin{array}{l}1 \\
5 \\
9\end{array}$ & $\begin{array}{r}385 \cdot 3 \\
6 \cdot 4 \\
12 \cdot 2\end{array}$ & $\begin{array}{c}31 \cdot 6^{* * *} \\
- \\
-\end{array}$ & $\begin{array}{r}371 \cdot 2 \\
10 \cdot 2 \\
5 \cdot 1\end{array}$ & $\begin{array}{l}71 \cdot 2 * * * \\
2 \cdot 0 \\
-\end{array}$ \\
\hline
\end{tabular}

Significance levels: * $5 \% ; * * 1 \% ; * * * 0 \cdot 1 \%$. 
values and Durrant and Tyson analysed the log. plant weights using methods of Hayman (1954a) and Jinks (1954). Hayman's analysis gave $\left(b_{1}\right)$ highly significant which agrees with the present analysis. The $W_{r} / V_{r}$ graph gave a line of unit slope and the $\left(W_{r}+V_{r}\right) / P_{r}$ correlation was virtually complete $\left(r=-0.96\right.$ and -0.94 for $\mathrm{F}_{1}$ and $\mathrm{F}_{2}$ respectively). The data also give highly significant $2 \bar{a}_{c}$ values (tables $9,10,11$ ) using log. plant weight. When the $W_{r}$ and $V_{r}$ values are recalculated using only the estimated $a_{c}$ values inserted in the diallel table, the points fall on a line of unit slope which is in the same position as the line obtained using the original data.

Both $M$ and $S$ have small values of $a_{c}$ in the $\mathrm{F}_{1}$ so that the deviations from the mid-parent values in each of their arrays are determined by the other parents, mainly the two linseed varieties $R$ and $D$. Since the $a_{c}$ values of $R$ and $D$ are positive and these varieties have the largest parental values they are also dominant, and $M$ and $S$ which have the smallest parental values are recessive. Consequently a parent may be characterised by whether it is dominant or recessive and whether the dominance or recessiveness is mainly determined by itself or by the other parents. In the $\mathrm{F}_{2}$ the $a_{c}$ value of $S$ is relatively high and would mean, were it significant, that the dominance of the other parents in crosses with $S$ are largely determined by $S$.

\section{(ii) $\mathrm{F}_{1}$ weights at birth and at 100 days in a diallel cross with rats}

Jinks and Broadhurst (1963) showed that at both times the $W_{r} / V_{r}$ graphs approximated to lines of unit slope but there was a reversal of dominance, line 6 having most dominant genes at birth and the most recessive genes at 100 days, line 3 having the most recessive at birth and the most dominant at 100 days. The negative correlation of $\left(W_{r}+V_{r}\right)$ at the two times is virtually complete, $r=-0.97$. As far as the analysis of reciprocal differences was taken (paper I) there was considerable scatter on the $W_{\odot} / W_{\delta}$ graphs, indicating large reciprocal differences, but significance was obtained at 100 days only, and using the more critical test mentioned here, both alpha and beta are significant. The $b_{g}$ values were positively correlated at the two times but the $a_{g}$ values were reversed, $r=-0 \cdot 75$. It could be that the reversal in alpha inheritance, i.e. the reversal of maternal effects at the later age, is responsible for the reversal in dominance shown by the $W_{r} / V_{r}$ graphs, a relation which may show up when the alpha reciprocal means and differences are compared. There was also a large negative $b_{g}$ value in the joint analysis at 100 days which was interpreted as being due to the milk yield of the parents.

Before analysing the reciprocal means a further examination of the reciprocal differences is necessary. The $b_{g}$ values (paper I) at 100 days are highly negatively correlated with the parents, $r=-0.94$, implying that larger parents are more dominant, and smaller parents are more recessive, in their female arrays since there are large positive and negative values of $b_{g}$. If interpreted in terms of maternal effects the negative correlation could be due to such causes as differences in litter size, differential response of $F_{1}$ families to maternal environments including milk yield, low correlation between parental weights within the diallel table and weight of parents crossed possibly because of reversal of effects, or milk yield not being positively correlated with parental weights. It could be, in fact, that the high $\bar{b}_{g}$ value in the joint analysis is due to the high correlation of $b_{g}$ with $P$. $k$ is -0.029 and an estimated value for $b_{g}$ assuming it to be entirely due to 
correlated beta, calculated from $k \Sigma P^{\prime 3} / 2 \Sigma P^{\prime 2}$, is $-0 \cdot 37$, which is fairly close to -0.30 (paper I). $b_{g}$ calculated from beta alone is positive, 0.15 (paper I); calculated from alpha alone it is -0.12 (not -0.06 as given in paper I) which is probably a reflection of correlated beta. Therefore the high negative $b_{g}$ value is reasonably interpreted as being due to correlated beta which

TABLE 12

Rat weight at birth; estimates

\begin{tabular}{|c|c|c|c|c|c|c|}
\hline \multirow[b]{2}{*}{ Array } & \multirow[b]{2}{*}{$a_{c}$} & \multirow[b]{2}{*}{$b_{c}$} & \multicolumn{2}{|c|}{ Joint } & \multicolumn{2}{|c|}{ Joint } \\
\hline & & & $2 \bar{a}_{c}$ & $b_{c}$ & $a_{c}$ & $b_{c}$ \\
\hline 1 & $-14 \cdot 7$ & 0.050 & - & 0.086 & -24.9 & -0.295 \\
\hline 2 & $16 \cdot 3$ & 0.141 & - & $0 \cdot 103$ & $-14 \cdot 3$ & 0.287 \\
\hline 3 & -10.4 & 0.070 & - & $0 \cdot 109$ & 83.9 & 0.743 \\
\hline 4 & 20.7 & 0.018 & - & 0.025 & $18 \cdot 2$ & $-0 \cdot 142$ \\
\hline 5 & $-21 \cdot 3$ & $-0 \cdot 110$ & - & $-0 \cdot 127$ & $-22 \cdot 3$ & -0.075 \\
\hline 6 & $16 \cdot 1$ & $-0 \cdot 169$ & - & -0.196 & $31 \cdot 2$ & -0.518 \\
\hline $2 \bar{a}_{c}$ & $2 \cdot 2$ & - & $6 \cdot 4$ & - & $23 \cdot 8$ & - \\
\hline
\end{tabular}

means that there is no simple and direct overall maternal effect, confirming the conclusions of Jinks and Broadhurst, but there is a more involved interplay between the $F_{1}$ genotypes and maternal characteristics and effects, or there are dominance changes in the maternal and paternal environments. This makes the comparison of reciprocal differences and dominance deviations more difficult.

TABLE 13

Rat weight at 100 days; estimates

\begin{tabular}{|c|c|c|c|c|c|c|}
\hline \multirow[b]{2}{*}{ Array } & \multirow[b]{2}{*}{$a_{c}$} & \multirow[b]{2}{*}{$b_{c}$} & \multicolumn{2}{|c|}{ Joint } & \multicolumn{2}{|c|}{ Joint } \\
\hline & & & $2 \bar{a}_{c}$ & $b_{c}$ & $a_{c}$ & $b_{c}$ \\
\hline 1 & -17.4 & 0.049 & - & $0 \cdot 165$ & $-126 \cdot 6$ & -1.018 \\
\hline 2 & $17 \cdot 1$ & 0.067 & - & -0.032 & 37.9 & -0.278 \\
\hline 3 & $17 \cdot 3$ & -0.434 & - & -0.293 & $45 \cdot 8$ & 0.393 \\
\hline 4 & $20 \cdot 7$ & -0.246 & - & -0.233 & $24 \cdot 2$ & 0.032 \\
\hline 5 & $8 \cdot 8$ & 0.139 & - & 0.038 & $-6 \cdot 2$ & 0.272 \\
\hline 6 & 19.5 & 0.425 & - & 0.355 & 1.6 & $0 \cdot 600$ \\
\hline $2 \bar{a}_{c}$ & $22 \cdot 0$ & - & $18 \cdot 8$ & - & $-7 \cdot 8$ & - \\
\hline
\end{tabular}

Alpha and beta estimates of the reciprocal means are given in tables 12,13 and the analyses of variance in table 14 where both joint estimates are significant. In each case alpha takes out a greater sum of squares than beta. The correlation coefficients between the estimates at birth and 100 days in table $15(a)$ show, unlike that for the $\left(W_{r}+V_{r}\right)$ values, no overall reversal in dominance; the reversal shown by beta alone, which contributes less to the dominance deviations, disappears in the joint analyses. The two sets of alpha values are in close agreement except those of parents 3 and 5 which have substantial reversals. The difference between the two forms of analysis may therefore be one of degree. The phenotype analysis is picking out a pattern of dominance deviations which is fairly uniform over the two times; the $\left(W_{r}+V_{r}\right)$ analysis is assessing the distribution of dominant genes at the two times on the assumption there are no gene interactions, or upsets due to large reciprocal differences, hidden in the $W_{r} / V_{r}$ graph. 
The correlations between reciprocal means and differences are given in table $15(b)$. At birth they are small. At 100 days alpha reciprocal differences, $a_{g}$, are significantly correlated with alpha dominance deviations, $a_{c}$,

TABLE 14

Rat diallel; analyses of variance

(i) $\begin{aligned} & 2 \bar{a}_{c} \\ & a_{c}^{\prime} \\ & \text { Residual }\end{aligned}$

(ii) Beta Residual

(iii) Beta plus $2 \bar{a}_{c}$ Residual

(iv) Alpha plus beta Residual

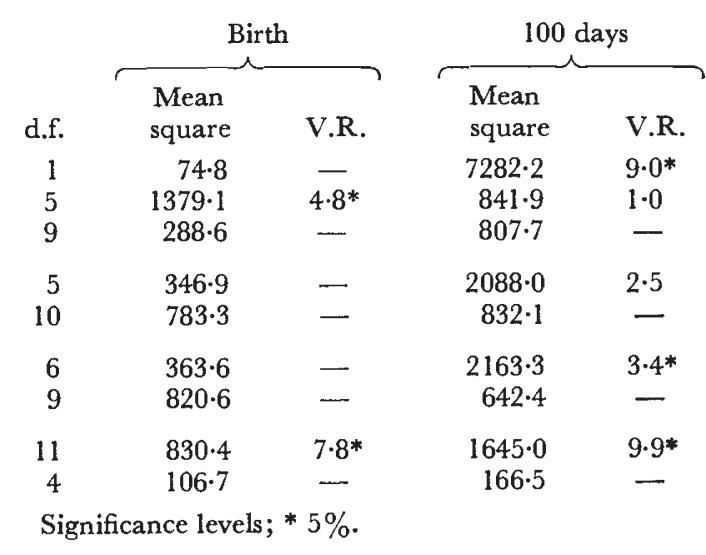

the negative value implying factors affecting the paternal arrays are responsible for part of the dominance deviations. Since the $a_{c}$ values are positively correlated at the two times the reversal of alpha reciprocal differences at

TABLE 15

Correlation and regression coefficients from rat diallel

$\begin{array}{lrr}\text { (a) Birth/100 days } & \begin{array}{c}\text { Correlation } \\ \text { coefficient }\end{array} & \begin{array}{r}\text { Regression } \\ \text { coefficient }\end{array} \\ \text { Alpha } & 0.62 & - \\ \text { Beta } & -0.62 & - \\ \text { Joint: } & & - \\ \quad \text { alpha } & 0.51 & - \\ \text { beta } & 0.13 & \\ \text { (b) Reciprocal means/differences } & & 0.26 \\ \text { At birth } & & 0.00 \\ \quad \text { Alpha } & 0.18 & 1.35 \\ \text { Beta } & 0.00 & -0.07 \\ \text { Joint: } & & \\ \quad \text { alpha } & 0.32 & -0.32 \\ \text { beta } & -0.05 & 0.14 \\ \text { At 100 days } & & \\ \text { Alpha } & & \\ \text { Beta } & -0.82 & -0.91 \\ \text { Joint: } & 0.69 & -0.49 \\ \quad \text { alpha } & & \\ \quad \text { beta } & -0.78 & \end{array}$

the two times mentioned above cannot be responsible for the reversal of dominance on the $W_{r} / V_{r}$ graph but could be responsible for reciprocal differences seemingly to be affecting the paternal, and not the maternal, 
arrays. When $a_{c}$ values are estimated from the $a_{g}$ values, using the regression coefficient, -0.32 , in table $15(b)$, and are subtracted from the diallel table, the reversal of the $\left(W_{r}+V_{r}\right)$ values at the two times remains. The beta correlation, table $15(b)$, is positive but not significant and the regression coefficient is small. In the joint analysis neither correlation is significant, although that of alpha is nearly so; alpha, and probably beta, have inflated regression coefficients.

Since there are substantial correlations at 100 days, estimated $a_{c}$ and $b_{c}$ values were calculated from the regression coefficients in table $15(b)$ and used to determine how far the reciprocal differences might be responsible for the dominance deviations. This was done for alpha, beta, joint alpha and beta, and joint alpha and beta with $b_{g}$ transferred to alpha. Estimated alpha took out two thirds of the mean square for $a_{c}^{\prime}$, beta accounted for one third of the mean square for $b_{c}$, in table 13 , and the others gave no fit. The negative

Table 16

Competition diallel

Species, $P,\left\{\begin{array}{cccccc}1 & \multicolumn{4}{c}{\begin{array}{c}\text { Associated species, } P_{8} \\ \text { Species } \\ \text { totals }\end{array}} \\ 2 & y_{11} & y_{12} & y_{13} & y_{14} & y_{1} . \\ 3 & y_{21} & y_{22} & y_{23} & y_{24} & y_{2} . \\ 4 & y_{31} & y_{32} & y_{33} & y_{34} & y_{3 .} . \\ & y_{41} & y_{42} & y_{43} & y_{44} & y_{4} .\end{array}\right.$

Associated species totals

$\begin{array}{llllll}y .8 & y .1 & y_{.2} & y_{.3} & y_{.4} & y . .\end{array}$

correlation of $\left(W_{r}+V_{r}\right)$ at the two times remained in each case. In the phenotype analysis therefore there is evidence that a good portion of the dominance deviations at 100 days could be due to reciprocal differences, presumably maternal effects which affect the parents and $F_{1}$ families differently. If the negative correlation in the $\left(W_{r}+V_{r}\right)$ analysis is due to reciprocal differences these occur erratically within reciprocal arrays or, if of the alpha or beta type, affect both male and female arrays to more or less the same extent. In a recent paper van Cortmerssen and Beardmore (1966) describe a reversal in dominance at two times of development in both reciprocals of a cross between two inbred lines of mice.

\section{Non-GeNetiG DiAllel tables}

Formulae for genetic reciprocal differences were modified for the analysis of competition diallels in paper I. Formulae for genetic reciprocal means can be applied directly to competition diallels, and it is only necessary here to interpret alpha and beta competition. A competition diallel, with slightly altered heading, is reproduced in table 16 where $y_{r s}$ is the weight of the species $P_{r}$ when grown with the associated species $P_{s}$. The mean yield of any two species grown separately, hereafter called the mid-species value, subtracted from their mean yields when grown together carried out over all pairs of species gives a table of $c_{r s}$ values similar to $1(b)$. A species has alpha competition if the mixture means involving that species are increased or 
decreased by a constant amount over the means of the respective pairs of species grown by themselves. A species has beta competition if the mixture means of that species are increased or decreased in proportion to the values of the associated species grown by themselves. Taking for example a small species, if the mixture means to which it contributes are equal to the midspecies values concerned there is neither alpha nor beta competition, but if the mixture means tend to the larger species by a constant amount this gives a positive value of $a_{c}$ for that species. If the mixture means tend to the other species in proportion to the values of the other species grown alone, a relatively large positive, or small negative, value of $b_{c}$ is obtained, i.e. in terms of the genetic diallel the species is recessive. Since members of each mixture are identifiable and can be compared with their respective species grown alone $a_{c} / a_{g}$ and $b_{c} / b_{g}$ correlation studies are not so useful as in genetic diallels.

Combining reciprocal means and differences,

$$
y_{r s}=\frac{1}{2}\left(P_{r}+P_{s}\right)+c_{r s}+\frac{1}{2} g_{r s} .
$$

The change in species $P_{r}$ due to alpha when grown with the other species is

$$
a_{r}=a_{c r}+\frac{1}{2} a_{g r},
$$

and the change in the associated species due to alpha when grown with $P_{r}$ is

$$
a_{\delta}=a_{c r}-\frac{1}{2} a_{g r} \text {. }
$$

Similarly beta contribution of species $P_{r}$ is

$$
b_{r}=b_{c r}+\frac{1}{2} b_{g r},
$$

and the beta contribution of the associated species when grown with $P_{r}$ is

$$
b_{s}=b_{c r}-\frac{1}{2} b_{g r} \text {. }
$$

On the $W_{r} / W_{\delta}$ graph, equivalent to the $W_{R} / W_{C}$ graph of paper I, all species are at $W_{r}=0, W_{\delta}=V_{P}$, denoted $\mathrm{Z}$ (paper $\mathrm{I}$ ), when there is no competition in reciprocal means or differences. Competition affecting only the reciprocal means spreads the points along a line of unit slope as in genetic diallels except that it passes through $\mathrm{Z}$ instead of the origin.

\section{Summary}

1. Formulae are set out for analysing reciprocal means of genetic diallel crosses in terms of alpha and beta inheritance.

2. Alpha measures individual parental contributions to uniform dominance deviations (potence) along their respective arrays. Beta measures the amount by which dominance changes with respect to the parental differences for each parental array (potence ratio). Both are related to alpha and beta inheritance of the reciprocal differences.

3. The analysis may be used to examine the phenotypic pattern, to determine how far uniform reciprocal differences are responsible for dominance deviations and for examining data wherein classical gene differences are not necessarily inferred.

4. The formulae are also applicable to competition diallels.

5. Analysis of a diallel cross of genotrophs and genotypes of Linum (Durrant and Tyson) gave significant alpha inheritance. 
6. A diallel cross of rats (Jinks and Broadhurst) gave significant joint alpha and beta inheritance at birth and 100 days, and evidence that some of the dominance deviations at 100 days was due to maternal effects, but changes in dominance at the two times could not be directly associated with the significant changes in reciprocal differences.

\section{REFERENGES}

AlLARD, R. w. 1956. The analysis of genetic-environmental interactions by means of diallel crosses. Genetics, 41, 305-318.

DURrant, A. 1965. Analysis of reciprocal differences in diallel crosses. Heredity, 20, 573-607. DURRANT, A., AND TYSON, H. 1964. A diallel cross of genotypes and genotrophs of Linum. Heredity, 19, 207-227.

EVANS, G. M., DURRANT, A., AND REES, H. 1966. Associated nuclear changes in the induction of flax genotrophs. Nature, 212, 697-699.

GRIFFING, B. 1956. A generalised treatment of the use of diallel crosses in quantitative inheritance. Heredity, 10, 31-50.

hayman, B. I. 1954a. The analysis of variance of diallel tables. Biometrics, 10, 235-244.

hayman, B. I. 1954b. The theory and analysis of diallel cross. Genetics, 39, 789-809.

JINKs, J. L. 1954. The analysis of continuous variation in a diallel cross of Nicotiana rustica varieties. Genetics, 39, 767-788.

JINKS, J. L., AND BROADHURST, P. L. 1963. Diallel analysis of litter size and body weight in rats. Heredity, 18, 319-336.

MCGILCHRIST, C. A. 1965. Analysis of competition studies. Biometrics, 21, 975-985.

VAN OORTMERSSEN, G. A., AND BEARDMORE, J. A. 1967. An age factor affecting variance of a behavioural character in $F_{1}$ hybrids between inbred lines of the house mouse. Experientia, 23, 1-7.

WIGAN, L. G. 1944. Balance and potence in natural populations. 7. Genetics, 46, 150-160. 